\title{
Value sets of functions over finite fields
}

\section{by}

\author{
S. D. Cohen (Glasgow)
}

1. Introduction and general results. Let $F$ be a finite field of order $q$ and characteristic $p$. Where necessary adjoin $\infty$ to $F$ as a possible value of a variable or function in the obvious way (see [2], §4). For any rational function $f=f_{1} / f_{2}$ in $F(x)$, where $f_{1}$ and $f_{2}$ are co-prime polynomials, define $V(f)$ to be the set of values taken by $f$ in $F$ and $\operatorname{deg} f$, the degree of $f$, to be $\max \left(\operatorname{deg} f_{1}, \operatorname{deg} f_{2}\right)$.

Our chief object in this paper is to discuss the extent to which a funetion $f$ of bounded degree is determined by $V(f)$. More precisely, we consider when $V(g) \subseteq V(f)$ can hold for two functions $f$ and $g$. In fact, we solve the problem completely for functions $f$ of degree not exceeding 4 . For details of the results see $\$ 2$.

The remainder of this section is devoted to a summary of the various general results which bring together and extend work discussed by the author in [1] and [2] and by M. Fried in [6], [8] and [10] and which form the abstract background from which the specifie functions of $\S 2$ will emerge.

Accordingly, let $h(x, y)$ be a polynomial in $x$ with coefficients in $F(y)$. We shall say that $h$ is $x$-soluble (in $Z^{\prime}$ ) if, for every $y$ in $F, h(x, y)=0$ is soluble with $x$ in $F$. For the application to value sets we shall set $h(x, y)$ $=f_{1}(x)-g(y) f_{2}(x)$, where $f=f_{1} / f_{2}$ and $g$ are rational functions in $F^{\prime}(x)$. (We shall frequently abuse notation and write $f(x)-g(y)$ for this polynomial or even for the numerator of the rational function $f(x)-g(y)$.)

Returning to the case of a general $h$, which need not even be irreducible, we outline a proof of the following result.

Proposirion 1.1. Let $h(x, y)$ be a separable polynomial of degree $m$ in $x$ with coefficients in $F(y)$ of degree $\leqslant n$. Let $h(x, y)=0$ have roots $x_{1}, \ldots, x_{m}$ in a splitting field $K$ over $F(y)$. Let $\bar{F}$ denote the algebraio closure of $F$ in $K$ and $G^{*}\left(K, F^{\prime}(y)\right)$, etc., the subset of the galois group $G\left(\mathbb{K}, F^{\prime}(y)\right)$ 
of $K$ over $F(y)$ comprising automorphisms whose restrictions to $\vec{F}$ fix preeisely IF. If

$$
G^{*}\left(K, F^{\prime}(y)\right)=\bigcup_{i=1}^{m} G^{*}\left(K, Z^{\prime}\left(x_{i}, y\right)\right),
$$

then $h$ is $x$-soluble. Conversety, if $q>o(m, n)$ and $h$ is $x$-soluble, then (1.1) holds.

Proof. We can assume that $h$ is square-free. For brevity, pat $\theta$ $=G\left(K, F^{\prime}(y)\right), G^{*}=G^{*}\left(K, W^{\prime}(y)\right), G^{*}(i)=G^{*}\left(\pi, F^{\prime}\left(x_{i}, y\right)\right), i=1, \ldots, m$, $G_{1}^{*}=\bigcup_{i=1}^{m} G^{*}(i)$. Also, for any $y_{0}$ in $F$, let $A\left(y_{0}\right)$ denote the conjugacy class in $Q$ which has the defining property of the Frobenius automorphism of some prime in $K$ dividing $y-y_{0}$. Certainly $A\left(y_{0}\right)$ exists; it is uniquely defined if $y-y_{0}$ is unramified in each $F\left(x_{i}, y\right)$, i.c. if $h\left(x, y_{0}\right)$ is square-free. Then, in fact, $A\left(y_{0}\right) \subseteq Q^{*}$ and indeed, if $y-y_{0}$ is unramified, then $A\left(y_{0}\right)$ $\subseteq G_{1}^{*}$ if and only if $h\left(x, y_{0}\right)=0$ is soluble in $F^{\prime}$ (see temma 3 of [1] and [2]). Moreover, even if $y-y_{0}$ is ramified, then $h\left(x, y_{0}\right)=0$ is soluble in $f^{\prime}$ provided $A\left(y_{0}\right) \subseteq G_{2}^{*}([2]$, p. 55, or [10], p. 223). Hence, (1.1) implies that $h$ is $x$-soluble. Oonversely, if $h$ is $x$-soluble, then, by the function field. analogue of the Cebotarev density theorem ([2], Lomma 2, [10], Proposition 2), for large $q, G^{*}=G_{1}^{*}$ and (1.1) holds.

Actually, in the last sentence of the above proof, it suffices to assume that $h$ is $x$-soluble with, at most, $k q^{8}$, say, exceptions where $0 \leqslant \delta<1$ (see [2], p. 59). Oonsequentily, the hypothesis of the second part can be weakened as in the following theorem.

THEOREM 1.2. In the sitnation of Proposition 1.1, suppose that $h$ is w-soluble except for at most $\mathrm{k} q^{\delta}$ values of $y$ in $F$, where $t_{i}>0$ and $0 \leqslant \delta<1$. If $q>o(m, n, \delta, t)$, then (1.1) holds and aotually $h$ is $x$-soluble in It. In partioular, if $f$ and $g$ are funetions of degree $\leqslant m, n$, respeotively and $q>e$, then $|\nabla(g) \backslash V(f)|<k q^{\delta}$ implies that $V(g) \subseteq V(f)$.

The final assertion of Theorem 1.2 completely resolves a conjecture and a conditional result of Fried ([5], Oonjecture 2, [8], Oorollary 2). It should have been proved in [2] but was obscured there by our not taking $h(x, y)=f(x)-g(y)$; in fact, the discussion was equivalent to putting $h(x, y)=(f(x)-y)(g(x)-y)$ so that values $y_{0}$ of $y$ for which $g(x)-y_{0}$ were not square-free had to be lett ont of the considurations.

In discussing possiblo occurences of (1.1), it may bo convenient to separate the cases in which $h$ is irreducible (in $I F[x, y]$ ) or reducible, respectively. Alternative conditions for an irreducible $h$ to be $x$-soluble are provided in the next result (c.. [2], Lemma 4 and Theorem 3, [8], Proposition 1).

Proposirion 1.3. In the situation of Proposition 1.1, let h be irreduoible in $F^{\prime}(x, y)$. Suppose that $q>o(m, n)$. Then the following are equivalent:

(i) $h$ is $x$-soluble in $F$;

(ii) $h(x, y)=0$ has a unique solution $x$ in $F$ for all $y$ in $F$ for which the diseriminant of $h$ (as a polynomial in $x$ ) is non-zero;

(iii) $h(x, y)$ is absolutely irreducible in $F(x, y)$ but has no absolutely irredncible factors except $(x-z)$ in $k '(x, y, z)$, where $h(z, y)=0$.

Indeed, for any $q$, (iii) implies (i) and (ii).

Proof. We use the notation enployed in proving Proposition 1.1. Note that the condition that $h(x, y)$ be absolutely irreducible in $F[x, y]$ is equivalent to the condition that $F\left(x_{i}, y\right) \cap \bar{F}=F$ for all $i=1, \ldots, m$ (ci. (4.10) of [2]). Hence, Lemma 4 of [2] shows that (iii) is equivalent to each of (a) $G^{*}=G_{1}^{*}$, and (b) the $G^{*}(i)$ are pairwise disjoint. By Proposition 1.1, (i) and (iii) are equivalent as required, while it follows from Theorem 1.2 that (ii) $\Rightarrow$ (i). Finally, suppose (i) holds. Then (a) and (b) are true. Hence every member of $A\left(y_{0}\right)$ belongs to precisely one $G^{*}(i)$. By [1], Lemma 5 , if $h\left(x, y_{0}\right)$ is square-free, then $h\left(x, y_{0}\right)=0$ has a unique solution in $f$. This completes the proof.

Note. It will follow from the examples of Theorem 2.1 (II) below that the exceptional $y$ in (ii) may definitely give rise to multiple solutions of $h(x, y)=0$ in $F$. (Thus some modification appears to be necessary in statement (2.12) of [8].)

Condition (1.1) for $h$ to be $x$-soluble is, at first sight, a very restrictive one. Indeed, it implies that $G=G\left(K, F^{\prime}(y)\right)$ is admissible in the following sense: $G$ can be represented as a permutation group on $(1, \ldots, m)$ and is a cyclic extension of a normal subgroup $\hat{G}$; moreover, if $G^{*}$ is the subset of $G$ every member of which generates $G \mid \hat{\theta}$, then $G^{*}=\bigcup_{i=1}^{m} G^{*}(i)$, where $G^{*}(i)$ denotes the stabilizer of $i$ in $G^{*}$. Indeed, for $h$ to be irreducible (and so absolutely irreducible, by Proposition 1.3 (iii)), an admissible $G$ has additionally to be transitive, and, since $F \neq \bar{F}$, the cyclic extension $G / \hat{G}$ must be non-trivial. (In the irreducible case, Fried, [11], p. 153, has given at description of an admissible $G$ corresponding to Proposition 1.3.)

Accordingly, in order to find all $x$-soluble $h$ of given degree $m$ in $x$, it is tirst necessary to find all admissible $G$ contained in the symmetric group $S_{m}$. This is straightforward for $m \leqslant 4$; there are two non-trivial possibilities with $G$ transitive and one with $G$ intransitive; in effect, these are dealt with in $\S \S 5-7$ below. More generally, the known examples of permutation polynomials, namely cyclic and Chebycher polynomials (see [9]), indicate that $\hat{G}$ may be a cyclic or metacyclic group. But there are other possibilities even when $G$ is transitive. Indeed, $\hat{G}$ need not even be solnble, as shown by the following example pointed out to the author 
by J. Saxl, in which $m=28$. Take $G=\operatorname{PrL}(2,8), \hat{G}=\operatorname{PGL}(2,8)$ so that $|G|=1512, \hat{G}$ is simple and $G / \hat{G}$ is cyclic of order 3 . As for the intransitive (reducible) case, Fried ([10], pp. 211, 227) has announced examples (with $h(x, y)=f(x)-g(y), f, g$ polynomials) which imply the existence of admissible $G$ with $G / G$ trivial (so that $\vec{F}=F$ ).

Having found an admissible $G$, we would next like to find all $h$ (if any) for which $G=G(K, F(y))$ (in the obvious correspondence). In the irreducible case, this includes what Fried [11] has called the "general Schur problem" and is very difficult. For general $h$ we give a solution only in the ease that the total degree of $h$ (in $x$ and $y$ ) does not exceed 3 (\$ 8). However, if $h(x, y)$ is of the form $f(x)-g(y)$, we can invoke properties of the discriminant and, in this way, obtain a complete solution provided $\operatorname{deg} f \leqslant 4$. These are the results listed in $\S 2$ and proved in $\$ \$ 3-7$.

Finally, in $\$ 9$, we shall consider some non-trivial examples of sets of functions $\left\{f_{i}\right\}$ which cover $F$, i.e. for which $\bigcup V\left(f_{i}\right)=F$.

2. Results on value sets. We describe here our main results on the existence of pairs of function $f(x), g(x)$ in $F^{\prime}(x)$ for which $V(g) \subseteq V(f)$.

Define a permutation function $P$ over $F$ to be one for which $V(P)$ $=\pi$. Then, trivially, $V(g) \subseteq \nabla(P)$ for any function $g$. Now obviously a non-singular, linear fractional transformation $L$ in $F(x)$ is a permutation function. However, there are others, e.g. the monomials $x^{n}$ provided $(n, q-1)=1$ and the Chebychev polyuomials $T_{n}$ for certain values of $n$, see [9]. These can be included in a more general class of functions of the form $f=\hat{f}(Q)$ for which $V(f)=V(\hat{f})$. The main result, which follows, shows that, in addition to such functions, there are some interesting pairs of functions $(f, g)$ with $\operatorname{deg} f \leqslant 4$ for which $V(g) \subseteq V(f)$. In its statement and throughout we use the following notation. $L$ denotes a nonsingular linear fractional transformation; $P$ is a permutation function; $\lambda$ is an arbitrary non-square in $F^{i}$; $F^{i}$ denotes the field of order $q^{i}$.

Theorsi 2.1. Let $f, g$ be rational functions in $E^{\prime}(x)$. Then $V(g) \subseteq V(f)$ if either (I) or (II) below holds.

(I). $f=\hat{f}(Q), g=\hat{f}(R)$ for some $\hat{f}, Q, R$ in $W^{\prime}(x)$ with

$$
\nabla(\hat{f}(Q))=V(\hat{f})
$$

In partioular, (2.1) is satisfied whenever $Q$ is a permutation funotion and $\hat{f}$ is any function.

(II). $p(=\operatorname{char} F)>3$ and $f=L \circ f^{*} \circ P, g=L \circ g^{*} \circ R$, where $L, P$ and $R$ are in $F(x)$ and $f^{*}$ and $g^{*}$ are one of the following pairs:

(i) $f^{*}(x)=x^{3}-3 x+2, g^{*}(x)=4 /\left(3 \lambda x^{2}+1\right)$;

(ii) $f^{*}(x)=x^{3}-3 \lambda x$,

$$
g^{*}(x)=2 \lambda\left[\alpha\left(x^{2}+\lambda\right)+2 \beta \lambda x\right] /\left[\beta\left(x^{2}+\lambda\right)+2 \alpha x\right],
$$

where $(\alpha, \beta)$ is a chosen pair in $F \times F$ for which $(-3 \lambda)\left(\alpha^{2}-\lambda \beta^{2}\right)$ is a non-zero. square in $F$;

(iii) $q \equiv 1(\bmod 3)$ and

$$
f^{*}(x)=\left(x^{4}+4 x^{3}\right) /(8 x-4), \quad g^{*}(x)=\mu x^{3}
$$

where $\mu$ is any non-oube in $F$;

$$
\text { (iv) } f^{*}(x)=x^{4}+4 x^{3} \text {, }
$$$$
g^{*}(x)=\left\{\begin{array}{l}
108 \mu x^{3} /\left(\mu x^{3}-1\right)^{2}, \quad \text { if } \quad q \equiv 1(\bmod 3), \\
108\left(x^{2}+3\right)^{3} /\left[v(x+\sqrt{-3})^{3}-\nu^{-1}(x-\sqrt{-3})^{3}\right]^{2}, \quad \text { if }
\end{array}\right.
$$

$$
q \equiv-1(\bmod 3),
$$

where $\mu$ is any non-cube in $F$ and $\nu$ is any non-oube in $F^{2}$ whose conjugate over $I$ is $\pm \nu^{-1}$ $3 *$

(v) $\alpha \neq 0, \frac{1}{4}, 1$ and $f^{*}(x)=\left[\left(x^{2}+3 a-3\right)^{2} / 4(2 x+3)\right]+3 \alpha-1$,

$g^{*}(x)=\left\{\begin{array}{l}\left(\mu^{2} x^{6}+a^{3}\right) / \mu x^{3}, \quad \text { if } \quad q=1(\bmod 3), \\ a^{3 / 2}\left[\nu(x+\sqrt{-3})^{6}+\nu^{-1}(x-\sqrt{-3})^{6}\right] /\left(x^{2}+3\right)^{8}, \quad \text { if }\end{array}\right.$

$$
q \equiv-1(\bmod 3)
$$

where $\mu$ is any non-cube in $F$ and $y$ is a non-cube in $F^{2}$ whose conjugate over $\mathbb{F}^{T}$ is $\nu^{-1}$ or $-\nu^{-1}$ according as a is, or is not, a square in $F$, respectively.

Conversely, suppose that $\operatorname{deg} f \leqslant 4, \operatorname{deg} g \leqslant n$ and that $q>o(n)$ with $p>3$. Then $V(g) \subseteq \nabla(f)$ implies that either $(\mathrm{I})$ or $(\mathrm{II})$ holds.

Remarks. (a) That (I) implies $\nabla(g) \subseteq V(f)$ is obvious. The sufficiency of (II) will emerge during the demonstration of the converse which, of course, is the harder task. (Note that, if $\operatorname{deg} f \leqslant 4$, then, in (II), we must have $P=L_{1}$.) Actually, the case in which $f$ and $g$ are cubic polynomials was partially considered by McCann and Williams [14] who showed that, if $q=p>7$, then $V(f)=\nabla(g)$ implied that $g=f(L)$ or $f=P$.

(b) For some values of $q$ we can explicitly simplify the form of the function $g^{*}$ in (ii). For if -3 or -1 is a square in $F$ we may choose $(\alpha, \beta)$ $=(1,0)$ or $(0,1)$, respectively. Thus we may take for $(2.2)$

$$
g^{*}(x)=\left\{\begin{array}{lll}
\lambda\left(x^{2}+\lambda\right) / x, & \text { if } & q \equiv-1(\bmod 3), \\
4 \lambda^{2} x /\left(x^{2}+\lambda\right), & \text { if } & q \equiv 1(\bmod 12)
\end{array}\right.
$$

(c) The $g^{*}$ of (iv) and (v) are in $F(x)$ despite the fact that, if $q \equiv$ $\equiv-1 .(\bmod 3)$, then $\sqrt{-3}$ and $v$ lie in $\mathbb{F}^{2} \backslash F$. In (iv), for example, $v$ could also be described as one of the $\frac{3}{4}(q+1)$ non-cubes in $\mathbb{1}^{2}$ which are $2(q+1)$-th roots of unity in $Z^{2}$. 
(d) Actually, in the excluded cases $\alpha=0,1,(v)$ remains valid but (after suitable linear transformations) reduces to (iii) and (iv), respectively.

(e) As regards (iii), since $\mu$ can be any non-cube in $F$, we also have $V\left(\mu g^{*}\right) \subseteq V\left(f^{*}\right)$. Indeed, we have

$$
V\left(f^{*}\right) \supseteq V\left(\mu x^{3}\right) \cup V\left(\mu^{2} x^{3}\right) .
$$

In particular $\left|V\left(f^{*}\right)\right| \doteqdot 3 q / 4,\left|\nabla\left(g^{*}\right)\right|=(q+2) / 3$.

(f) In case (II), the containments $V(g) \subseteq V(f)$ are all proper. This is apparent from (2.3) in case (iii). Otherwise, in cases (i) and (ii) we have, approximately, $|\nabla(f)|=2 q / 3,\left|\nabla\left(g^{*}\right)\right|=\frac{1}{2} q$, while in cases (iv), $(\mathrm{V}),\left|V\left(f^{*}\right)\right|$ $=5 q|8,| \nabla\left(g^{*}\right) \mid=\frac{1}{3} q$.

(g) If the degree of $f$ is allowed to exceed 4, it remains to describe what other exceptional cases require to be added to (II). Certainly, if $f(x)-$ $-g(y)$ is reducible then, as mentioned in $\S 1$, Fried [10] has asserted that there are algebrbie number fields $K$ and polynomiats $f$ and $g$, defined. over $K$ and not linearly related such that $V(f(\bmod p))=V(g(\bmod p))$ for almost all prime ideals $\mathfrak{p}$ of $K$. On the other hand, if $f(x)-g(y)$ is irreducible, then, although there are additional admissible possibilities (in the sense of $\xi 1$ ) for the galois group of $h(x, y)$, these may never be realised by $h$ of the form $f(x)-g(y)$.

Of course an explicit classifieation of all functions satisfying $(\mathrm{I})$ is desirable. We provide such for $\operatorname{deg} f \leqslant 4$. First wo describe the permatation. functions. Wo show that the only non-trivial ones are of degree 3. (Of course, the non-existence of permutation polynomials of degrees 2 and 4 is well known.) In particular, we show that there is a class of permutation functions of degree 3 which includes no polynomials.

TeEOREM 2.2. Let $f$ be a permutation funetion of degree $\leqslant 4$. Suppose that $q>0$ (absolute) and $p>3$. Then $f=L$ or $f=L_{1} \circ f^{*} \circ L_{2}$, where

$$
f^{*}(x)=\left\{\begin{array}{l}
x^{3}, \quad \text { if } \quad q \equiv-1(\bmod 3), \\
\left(x^{3}+3 \lambda x\right) /\left(3 x^{2}+\lambda\right), \quad \text { if } \quad g \equiv 1(\bmod 3) .
\end{array}\right.
$$

Next, wo show that (I) may hold with $Q \neq P$ even when $H(f) \leqslant 4$. It is enough to suppose that $g=\hat{f}$ so that $V(f)=V(g)$.

THEoriem 2.3. Suppose that $\operatorname{deg} f \leqslant 4$ and $\operatorname{deg} g \leqslant n$. If $g>o(n)$ and $p>3$, then $V(f)=V(g)$ if and only if $g=f(P)$ or $f=x \circ f^{*} 0 L_{1}, g$ $=L_{\circ} g^{*} \circ P$, where $f^{*}$ and $g^{*}$ are one of the following pairs:

(i) $f^{*}(x)=x^{4}, g^{*}(x)=x^{2}$ and $q \equiv-1(\bmod 4)$;

(ii) $f^{*}(x)=\left(x^{4}+\lambda\right) / x^{2}, g^{*}(x)=\left(x^{2}+\lambda\right) / x$;

(iii) $f^{*}(x)=\left(x^{2}+\lambda\right)^{2} / 2\left(x^{3}-\lambda x\right), g^{*}(x)=\left(x^{2}+\lambda\right) / \infty$.

3. Auxiliary results. When $h$ has degree $\leqslant 4$ (in $x$ ) some of the results of $\S 1$ can be rephrased in a manner involving its discriminant. In fact, if $h(x, y)=f(x)-g(y)$, we shail find that, by considering the shape of the discriminant, the functions $f$ and $g$ can be normalized, thereby greatly simplifying the argument.

Accordingly, let $h(x, y)$ be a square-free polynomial of degree $m(\geqslant 2)$ with coefficients in $F(y)$ and zeros $x_{1}, \ldots, x_{m}$ in a splitting field. Let $D_{h}(y)$ denoto the discriminant $a^{2 m-2} \prod_{i \neq j}\left(x_{i}-x_{j}\right)$ of $h$, where $a=a(y)$ is its leading coefficienti. Further, for any $f$ in $F(x)$, we shall also, without fear of ambiguity, use $D_{f}(y)$ to denote the polynomial $D_{f_{1}(y)-f_{2}(y)}(y)$ (in $F[y]$ ), where $f(x)=f_{1}(x) / f_{2}(x)$ and $f_{1}$ and $f_{2}$ are co-prime polynomials with $f_{1}$ monie. We summarise some relevant properties of $D_{f}$ which are due essentially to the fact that the extension $F^{\prime}(x, y)$ of $F^{\prime}(y)$, where $f(x)=y$, has genus 0 . They are actually valid for any field $F$ whose characteristic $>m$. In our case, assume $p>m$.

In the first place, $\operatorname{deg} D_{f} \leqslant 2 m-2$. Put $r_{\infty}=2 m-2-\operatorname{deg} D_{f}$. Suppose that $D_{f}$ has prime decomposition $\alpha \prod_{i=1}^{s} \mathscr{P}_{i}^{r_{i}}$ in $F[y]$ where $\alpha(\neq 0) \in F$ and the $\mathscr{P}_{i}$ are monic irreducibles. Formally adjoin a linear polynomial denoted (temporarily) by $\mathscr{P}_{\infty}$ which vanishes at $\infty$ and put $\mathscr{D}_{f}=\left(\prod_{i=1}^{8} \mathscr{P}_{i}^{r_{i}}\right) \mathscr{P}_{\infty}^{\tau_{\infty}}$. Befer to the set of ordered pairs of the form ( $\operatorname{deg} \mathscr{P}, r$ ) (with multiplicities) as included in the ramification data of $f$ over $F$. Its significance is as follows. Let $\gamma$ be any root of $\mathscr{P}_{i}(y)=0$ in $\bar{F}$, the algebraic closure of $F$. Let the zeros of $f_{1}-\gamma f_{2}$ in $\bar{F}$ have multiplicities $e_{1}, e_{2}, \ldots$, with the convention that, if $e_{\infty}=m-\operatorname{deg} f_{2}$ is non-zero, then $e_{\infty}$ is included. Then, of course, $\sum e_{j}=m$, but in fact, we also have $\sum\left(e_{j}-1\right)=r_{i}$. The collections $E\left(\mathscr{P}_{i}\right)=\left\{e_{1}, e_{2}, \ldots\right\}$ complete the ramification data of $f$. Note that $\mid E\left(\mathscr{P}_{i}\right)$ $=m-r_{i}$. Since $F(\gamma)=F(L(\gamma))$ for any $L$ in $F(x)$ and $\gamma$ in $\bar{F}$ (adjoining $\infty$ to $F$, if necessary), it is clear from the above interpretation of the ramification data, that it is preserved under compositions of the form $L_{1} \circ f \circ L_{2}$ with $L_{1}, L_{2}$ in $F(x)$. Further, if the pair $(1, r)$ is included in the ramification data, then by replacing $f$ by $L(f)$ for appropriate $L$, we can assume that $\mathscr{P}^{r}$ appears in $\mathscr{D}_{f}$, so that $f_{2}$ has prime decomposition of the form $f_{2}$ $=\beta P_{1}^{\alpha_{1}} P_{2}^{e_{2}} \ldots\left(e_{j}>0\right)$ where $\sum\left(e_{j}-1\right) \leqslant r$. In this situation, if $\operatorname{deg} P_{1}=1$, we can replace $f$ by $f(L)$ and assume that $\operatorname{deg} f_{2}=m-e_{1}$.

To complete the preliminaries we state a vital lemma, which follows immediately from a more general result of the author [3].

LemMna 3.1. Suppose that $r=2$ or 3 and $p>3$. Let $A$ and $B$ be rational functions in $F(x)$ with $A$ not an $r$-th power in $F(x)$. Suppose that $A(B)$ is an $r$-th power in $F(x)$. If $r=2$, then $A=Q A_{1}^{2}$, where $A_{1} \in F(x)$ and $Q$ is a polynomial of degree $\leqslant 2$ in $I[x]$. If $r=3$, then $A=L A_{1}^{3}$, where $L$, $A_{1} \in F(x)$. 
An explicit description of those $A, B$ for which $A(B)$ is an $r$ th power (for any $r$ ) is given in [3].

4. The quadratic case. If $\operatorname{deg} f=1$ then, of course, the results of $\S 2$ are trivial. The case $\operatorname{deg} f=2$ is disposed of in the following theorem.

Theorim 4.1. Let $h(x, y)$ in $F(x, y)$ have degree 2 in $x$ and degree $n$ in $y$. Suppose $q>o(n)$ and $p>2$. Then $h$ is $x$-soluble in $z^{\prime}$ if and only if it is reducible in $F^{\prime}(x, y)$. In particular, suppose $f$ and $g$ are functions in $F(x)$ with $\operatorname{deg} f=2, \operatorname{deg} g=n$. Then the following are equivalent:

(i) $\nabla(g) \subseteq V(f)$;

(ii) $g=f(R)$ for some $R$ in $F(x)$.

If also $\operatorname{deg} g=2$, then the following are each equivalent to (i) or (ii).

(iii) $V(g)=V(f)$;

(iv) $g=f(L)$;

(v) $D_{0}(y)=D_{f}(y) v^{2}(y)$, where $v(y) \in \Gamma(y)$.

Proof. Condition (iii) of Proposition 1.3 ain never hold if $m=2$ and the first part is clear. If $h(x, y)=f(x)-g(y)$, then reducibility of $h$ is equivalent to (ii) so that (i) and (ii) are equivalent. Finally, suppose that $\operatorname{deg} g=2$. The following implications are obvious: (ii) $\Leftrightarrow$ (iv) $\Rightarrow$ (iii) $\Rightarrow(\mathrm{i}) \Rightarrow$ (ii). Fence (i)-(iv) are equivalent. Moreover, (iv) $\Rightarrow(v)$ while (v) $\Rightarrow$ (iii) is an easy property of the discriminant.

5, Functions of degree 3. In the cubic case we nse Proposition 1.3 in the following form.

Propositron 5.1. Suppose, in Proposition 1.3, that $h$ is a oubic in $x$ and $p>3$. Then the following can be added to the list of equivalent conditions (i)-(iii):

\section{(5.1) (iv) $\quad D_{h}(y)=\lambda v^{2}(y)$, where $v(y) \in F(y)$.}

Proof. For a given $y$ in $T, h(x, y)$ has a unique zero (of multiplicity 1 ) in $F$ if and only if $D_{h}(y)$ is a non-square in $F$. Thus (iv) $\Rightarrow$ (ii) while (ii) $\Rightarrow$ (iv) (for large $q$ ) follows from a result of Perel'muter [1.8].

We now take $h(x, y)=f(x)-g(y)$ and proceed to prove the results of $\S 1$ with $\operatorname{deg} f=3$. Trivially, in this case, (I) of Theorem 2.1. occurs if and only if $Q=P$. We can assime $h$ ixreducible.

Suppose therefore that $V(g) \subseteq V(f)$. This property clearly survives the operation of replacing $f$ and $g$ by $L \circ f \circ L_{1}$ and $L \circ g \circ L_{2}$, respectively. By Proposition 5.1, $\lambda D_{f}(g(y))$ is a square in $F^{\prime}(y)$. Tence, by Lemma 3.1 . $\lambda D_{f}(y)$ is a square apart from a factor of degree at most 2. If $\operatorname{deg}\left(D_{f}(y)\right)$ $>2$, then $D_{f}(y)$ must have a square factor, while if $\operatorname{deg}(D(y))=2$, then certainly $\mathscr{L}_{\infty}^{2}$ divides $\mathscr{D}_{f}$, where now $\mathscr{L}_{\infty}$ denotes the infinite linear factor $\mathscr{P}_{\infty}$ of $\S 3$. Hence, in either case, $\mathscr{D}_{f}$ has a square factor. We use $\mathscr{L}, \mathscr{L}_{1}, \mathscr{L}_{2}$ to denote distinct linear polynomials (possibly $\mathscr{L}_{\infty}$ ) and $\mathscr{Q}$ to denoto an irreducible quadratic polynomial in $F[y]$ and consider the three possibilities for $\mathscr{D}_{f}$.

(a) $\mathscr{D}_{f}=\mathscr{L}_{1}^{2} \mathscr{L}_{2}^{2}$. As in $\S 3$, we may assume that, in fact, $\mathscr{L}_{2}=\mathscr{L}_{\infty}$ and that $f$ is a polynomial. Indeed, by linear transformations we may take $\mathscr{D}_{f}(y)=y^{2}$, whence $L \circ f \circ L_{1}=x^{3}$. So assume $f(x)=x^{3}$. Then $D_{f}(y)$ $=-27 y^{2}$ and hence -3 is a non-square in $F$ (i.e. $q \equiv-1(\bmod 3)$ ) and $f$ is a permutation polynomial.

(b) $\mathscr{D}_{f}=2^{2}$. In this case we may assume that $2(y)=y^{2}-\lambda$. It follows that, if $f=f_{1} / f_{2}$, then

$$
f_{1}(x)+\sqrt{\lambda} f_{2}(x)=\left(\alpha_{1}+\sqrt{\lambda} \alpha_{2}\right)\left(v_{1}(x)+\sqrt{\lambda} v_{2}(x)\right)^{3},
$$

where $\alpha_{1}, \alpha_{2} \in F$ and $v_{1}$ and $v_{2}$ are linear or constant polynomials in $F[x]$. Thus, replacing $f$ by $L \circ f \circ L_{1}$, where $L_{\perp}^{-1}(x)=\left(\alpha_{1} x+\lambda \alpha_{2}\right) /\left(\alpha_{2} x+\alpha_{1}\right)$, $L_{1}^{-1}=v_{1} / v_{2}$, we obtain

$$
f_{1}(x)+\sqrt{\lambda} f_{2}(x)=(x+\sqrt{\lambda})^{3}
$$

whence $f(x)=\left(x^{3}+3 \lambda x\right) /\left(3 x^{2}+\lambda\right)$. Accordingly, $D_{f}(y)=-108 \lambda\left(y^{2}-\lambda\right)^{2}$, -3 is a square in $F$ and $q \equiv 1(\bmod 3)$. Moreover, by Proposition 4.1, this $f$ is a permutation function.

(c) $\mathscr{D}_{f}=\mathscr{L}^{2} \mathscr{L}_{1} \mathscr{L}_{2}$ or $\mathscr{L}^{2} \mathscr{2}$. As before we may assume that $\mathscr{L}=\mathscr{L}_{\infty}$ and indeed that $f$ is a polynomial. In fact, by a linear transformation of $x$, we may take $f(x)=x^{3}-3 \eta x$, where $\eta=1$ or $\lambda$. Put $g(y)=2 \eta u$. Then $D_{f}(2 \eta u)=-108 \eta^{2}\left(u^{2}-\eta\right)$. Hence $(-3 \lambda)\left(u^{2}-\eta\right)$ is a square in $F(y)$ and

(5.2) $\quad \nabla(g) \subseteq S:=\left\{2 \eta \alpha:(-3 \lambda)\left(\alpha^{2}-\eta\right)\right.$ is a square in $\left.F\right\} \subseteq V(f)$.

Now, for the moment suppose $\eta=1$ and put $g_{0}(y)=4\left(3 \lambda y^{2}+1\right)^{-1}-2$. Then $D_{\rho_{0}}(2 y)=(-12 \lambda)(y-1) /(y+1)$ and evidently $V\left(g_{0}\right)=S$. Hence

$$
\nabla(g) \subseteq \nabla(f) \Leftrightarrow V(g) \subseteq V\left(g_{0}\right) \Leftrightarrow g=g_{0}(R),
$$

for some $R$ in $F(x)$, by Theorem 4.1 (ii). The necessity and sufficiency of (i) of Theorem 2.1 (II) follows.

Next suppose that $\eta=\lambda$ and that $\alpha$ and $\beta$ in $F$ are such that $(-3 \lambda)\left(\alpha^{2}-\lambda \beta^{2}\right)$ is a non-zero square in $F$. Put

$$
g_{0}(y)=2 \lambda\left[\alpha\left(y^{2}+\lambda\right)+2 \beta \lambda y\right] /\left[\beta\left(y^{2}+\lambda\right)+2 \alpha y\right] .
$$

Then $D_{g_{0}}(y)(2 \lambda y) /(-3 \lambda)\left(y^{2}-\lambda\right)$ is a square in $F(y)$. By comparing this with (5.2) and using the argument of the $\eta=1$ case, we see that $V(g)$ $\subseteq V(f) \Leftrightarrow g=g_{0}(R)$. To complete the proof, it remains to show that, if $g^{*}$ is also given by (5.3) with another pair $(\alpha, \beta)$, then $g^{*}=g_{0}(J)$. By Theorem 4.1 (iii) $-(\mathrm{v})$, this is so. 
6. Function of degree the ixredncible case. Wo suppose now that $h(x, y)=\sum_{i=0}^{\infty} h_{i} x^{i}\left(h_{4} \neq 0\right)$ is a quartic polynomial in $x$ with cocfficients in $I^{\prime}(y)$. Its classical cubic resolvent, namely

$$
x^{3}-h_{2}^{\prime} x^{2}+\left(h_{3}^{\prime} h_{1}^{\prime}-4 h_{0}^{\prime}\right) x-h_{3}^{\prime} h_{0}^{\prime}+4 h_{2}^{\prime} h_{0}^{\prime}-h_{1}^{\prime 2},
$$

where $h_{i}^{\prime}=h_{i} / h_{h}$, will be denoted by $\mathscr{R}_{h}(x, y)$. In the first instance wo suppose that $h$ is irreducible; thus, for example, Proposition 1.3 is applicable. Thecall that $T^{i}$ denotes the field of onder $q^{i}$.

Proposincon 6.1. In the situation of Proposition 1.1, suppose that $h$ has degree 4 in $x$ and is irreducible over $F(x, y)$ and that $p>3$. Then $h$ is $x$-soluble in $\mathbb{F}$ if and (when $q>c(n)$ ) only if $\mathscr{B}_{h}(x, y)$ is irreduoible in $F(x, y)$ but reduoible in $H^{13}(x, y)$. In partioular, if $h$ is x-soluble in $F$ and $q>e(n)$, then

(i) $h$ is $x$-soluble in $\mathbb{F}^{2}$ and

(ii) $D_{h}(y)=h_{4}^{6} D_{\mathscr{B}_{h}}(y)$ is a square in $F(y)$.

Proof. We use the notation of Proposition 1.1. Suppose $h$ is $x$-soluble in $F$. Since $h$ is irreducible, then $F \neq \bar{F}$ and $|G(K, F(y))|$ is divisible by 4 . Indeed, by Proposition 1.3 (iii), $|G(K, F(y))|$ is also divisible by 3 . In fact, since $G(K, F(y))$ is a cyclic extension of $G(K, \bar{F}(y))$, we must have $G(R, F(y))=A_{4}$ and $G(K, \bar{F}(y))=V:=\{1,(12)(34),(13)(24),(14)(23)\}$. Thus $\bar{F}=\bar{H}^{3}$. Accordingly (ii) holds and (i) follows from Proposition 1.3 (iii). Moreover, by [13], Theorem $43, \mathscr{R}_{h}$ is irreducible in $\mathbb{F}^{\prime}(x, y)$ but reducible in $F^{3}(x, y)$. Conversely, if this last fact holds then $G(K, F(y))$ $=A_{4}$ and $G\left(K, F^{3}(y)\right)=V$. But $A-V$ comprises only 3-cycles and so (2.1) is satisfied and consequently $h$ is $x$-soluble. This completes the proof.

From Proposition 6.1, the $x$-solubility of $h$ depends on the reducibility of the cubic $\mathscr{R}_{h}$. Accordingly, we need a result which follows easily from "Cardan's formulas" for the solution of a cubic equation (seo [12], p. 258).

Lemma 6.2. Let $\mathscr{R}(x)=x^{3}+a x+\beta$ where $\alpha, \beta$ (not both zero) belong to a field $\Omega$ of oharaoteristic $>3$. Suppose that the disoriminant $D$ of $\mathscr{D}$ is a square in $\Omega$. Then $\mathscr{R}(x)=0$ has one (and so all) solutions in $\Omega$ if and only if for ome shoice of the square root $\sqrt{D}$,

$$
\theta=\left\{-\frac{7}{2}(\beta+\sqrt{(D /-27)})\right\}^{1 / 3}
$$

belongs to $\Omega(\sqrt{-3}) \backslash\{0\}$.

We now specialise to the case $h(x, y)=f(x)-g(y)$, where $f$ has degree 4 , but still assume $f$ irreducible. As far as the results of $\$ 2$ are concerned, we now show that $V(g) \subseteq V(f)$ if and only if $f$ and $g$ are given by one of (iii)-(v) of Theorem 2.1 (II).
Assume then that $V(g) \subseteq V(f)$. By Proposition $6.1, D_{f}(g(y))$ is a square in $I^{\prime}(y)$ and so, by lemma 3.1., $D_{f}(y)$ (which las degree $\leqslant 6$ ) is also a square apart from a factor of degree at most 2. Indeed, a quick survey of the various yossibilities roveals that actually $\mathscr{D}_{f}$ is a square apart from a factor of degree at most 2 . We consider the various possibilities according to the factorisation of $\mathscr{D}_{j}$, using $\mathscr{L}, \mathscr{L}_{1}, \ldots, \mathscr{Q}, \mathscr{2}_{1}, \ldots, \mathscr{C}$, for distinct linear, quadratio and cubic irreducibles, respectively. As in $\$ 5$, we pass freely from $f$ and $g$ to equivalent pairs $L \circ f \circ L_{1}$ and $L \circ g \circ L_{2}$. Some preliminary observations are helpful. Tirst, if $\mathscr{g}$ divides $\mathscr{D}_{f}$ with, in the notation of $\$ 3, r=\ldots r_{s}=2,2$, thon $B(\mathscr{P})$ cun bo wither $\{1,3\}$ or $\{2,2\}$. Again if $\mathscr{L}_{1}$ and $\mathscr{L}_{2}$ both divides $\mathscr{D}_{f}$ and $\mathbb{E}\left(\mathscr{L}_{3}\right)$ and $\mathscr{Z}\left(\mathscr{L}_{2}\right)$ are either $\{2,2\}$ or $\{4\}$ (although possibly unequal), than replacing $f$ by $L_{\circ} \circ \circ L_{1}$ as appropriate, we get $f=\alpha f_{0}^{\prime \prime}$, where $M\left(f_{0}\right)=2$, so that for any $g$, certainly $\left|Q\left(\pi, F^{\prime}(y)\right)\right|$ $\leqslant 8$ which is impossible, granted that we are disenssing only the irreducible case meantione.

(a) ${ }^{(1)} \mathscr{L}^{3}$ divides $\mathscr{D}_{f}$. Using the ideas of $\& 3$ (as wready employed in $\S 5$ ), we may assumo that $\mathscr{L}=\mathscr{L}_{\infty}$ and, indeed, that $f$ is a polynomial with $\mathscr{D}_{f}=\mathscr{L}_{\mathrm{a}}^{2} \mathscr{L}_{2} \mathscr{E}_{\infty}^{3}$. Applying a linear transformation to $x$ and multiplying by a suitablo constant, wo may assume oven that $f(x)=x^{4}+4 x^{3}$. Put $u=g(y)$. The oubic resolvent of $f(x)-u$ is

$$
\not(x)=x^{3}+4 u x+16 u \text {. }
$$

Moreorex, sines $D_{f}(u) \ldots D_{\text {像 }}(u)=-256 u^{2}(u+27)$ is a square in $F(y)$, then $u=-X^{2}-27$ whero $R(y) \in F(y)$. By Lemma $6.2, \mathscr{R}(x)$ is reducible in $\mathbb{F}^{\prime \prime}(x, y)$ but not in $F(x, y)$ if and only if

$$
(2 / \sqrt{-3})\left\{(R+\sqrt{-27})\left(R^{2}+27\right)\right\}^{1 / 3} \quad \text { belongs to }
$$

$$
\mathbb{F}^{3}(\sqrt{-3}, y) \backslash F(\sqrt{-3}, y) \text {. }
$$

Suppose, for the moment that $q \equiv 1(\bmod 3)$ so that $\sqrt{-3} \in F$. Then ovidently $(6.2)$ holds if and only if

$$
(R-\sqrt{-27}) /(R+\sqrt{-27})=\mu S^{3}
$$

where $\mu$ is an non-ento in $H^{\prime}$ and $s^{\prime} \in H^{\prime}(y)$. Ilowever $(6.3)$ is equivalent to

$$
k=\sqrt{m-27}\left(\mu S^{3}+1\right) /\left(\mu S^{3}-1\right) .
$$

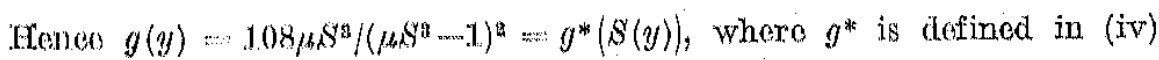
of Theorem 2.1. (Ir) (with $q$ : 1. (mod 3)).

Alternatively suppose that $q=-1$ (mod 3$)$ so that $F(\sqrt{-3})=F^{2}$. Wo roquiro (6.3) to hold with $\mu$ a nom-cube in $F^{2}$ and $S$ in $F^{2}(y)$ but $R$ (given by $(6.4)$ ) in $W^{\prime}(y)$. This occurs if and only if the product of $\mu S^{3}$

(i) Actrally, oaso (ii) cass be treated along with caso (c). 
and $\overline{\mu S^{3}}$, its conjugate over $F(y)$, is 1 . In fact, wo must have $\mu S^{3}$ $=\delta(T+\sqrt{-3})^{3} /(T-\sqrt{-3})^{3}$, where $\delta$ is a non-cube in $F^{2}$ such that $\delta \delta=\delta^{a+1}=1$ and $T \in F(y)$. This leads to

$$
\begin{aligned}
g(y) & =108 \delta\left(T^{2}+3\right)^{3} /\left(\delta(T+\sqrt{-3})^{3}-(T-\sqrt{-3})^{3}\right)^{2} \\
& =108\left(T^{2}+3\right)^{3} /\left(\nu(T+\sqrt{-3})^{3}-\nu^{-1}(T-\sqrt{-3})^{3}\right)^{2},
\end{aligned}
$$

where $\nu^{2}=\delta$ and $\nu$ is as described in (iv) of Theorem 2.1 (II); in particular $v \in \mathbb{F}^{2}$ since $\delta^{q+1}=1$. Elence $g(y)=g^{*}(T(y))$, as required. Since the steps are reversible, this completes the proof in this case.

(b) $\mathscr{L}_{1}$ and $\mathscr{L}_{2}$ divide $\mathscr{D}_{f}$ with $D\left(\mathscr{L}_{1}\right)=\mathbb{E}\left(\mathscr{L}_{\mathrm{a}}\right)=\{1,3\}$. In the usual way take $\mathscr{L}_{2}=\mathscr{L}_{\infty}$ and $\operatorname{deg} f_{2}=1$. A linear transformation in and multiplication by a coustant enable us to concentrate our attention on the function $f(x)=\left(x^{4}+4 x^{3}\right) /(4 x+a)$, where $\alpha(\in F) \neq 0,16$ (otherwiso $f$ is not in its lowest terms). Put $g(y)=u$ and let $\mathscr{R}(x)$ bo the resolvent cubic of $f(x)-u$. We have

$$
\mathscr{R}(x)=x^{3}+4(\alpha-4) u x+16 u(\alpha-u)
$$

and

$$
D_{f}(u)=D_{\mathscr{R}}(u)=256 u^{2}\left[-27(\alpha-u)^{2}-u(\alpha-4)^{3}\right]=256 u^{2} Q(u),
$$

say. Moreover, taking $\mathscr{R}$ as the polynomial (6.5) in Lemma 6.2, we have

$$
\theta=-2\{u[(\alpha-u)+\sqrt{(Q(u) /-27)}]\}^{1 / 3} .
$$

Now $Q(u)$ has a repeated factor (in $F(u)$ ) if and only if $\alpha=-2,4$. or 16. However, $a=16$ has been excluded. Moreover, if $\alpha=4$ and $\sqrt{Q(u)}$ is taken to be $\sqrt{-27}(4-u)$, then, in fact, $\theta=2[2 u(u-4)]^{1 / 3}$. But Lemma 3.1 with $r=3$ implies that $2 u(u-4)$ can never be a cube in $F^{3}(y)$ for any $g$ and so, by Lemma 6.2 and Proposition 6.1, we cannot have $V(g) \subseteq V(f)$. Next, putting $a=-2$, we obtain $Q(u)=-27(u-2)^{2}$. Since $D_{f}(u)$ is a square in $F(u)$, we must then have $\sqrt{-3} \in \mathbb{F}$, i.e. $q=1(\bmod 3)$. Wurther, taking $\sqrt{Q(u)}=\sqrt{-27}(u-2)$ in $(6.6)$, we require $(-32 u)^{1 / s}$ to bo in $I^{1 s}(y)$ but not in $F^{\prime}(y)$. Olearly, this is the case if and only if $u=2 \mu R^{g}$ for somo $R$ in $F^{\prime}(y)$ and non-cube $\mu$ in $F$, i.e. if and only if $u=2 g^{*}(R)$, where $g^{*}$ is given by (iii) of Theorem 2.1 (II). Conversely, for $f^{*}$ and $g^{*}$ as givon there, the above argument shows that $V\left(g^{*}\right) \subseteq V\left(f^{*}\right)$ for any $q$ (with $p>3$ ) and that actually, $\mathscr{D}_{f^{*}}=\mathscr{L}_{1}^{2} \mathscr{L}_{2}^{2} \mathscr{L}_{3}^{2}$, where $\mathbb{E}\left(\mathscr{L}_{1}\right)=\mathbb{D}\left(\mathscr{L}_{2}\right)=\{1,3\}$ and $E\left(\mathscr{L}_{3}\right)=\{2,2\}$ (since $\left.x^{4}+4 x^{3}-8 x+4=\left(x^{2}+2 x-2\right)^{2}\right)$.

To conclude this case, it suffices to show that $Q(u)$ cannot be squarefree. For suppose $Q(u)=-27(u-a)(u-b)$, where $a, b \in F^{2}$ with $a \neq b$. Then $(u-a) /(u-b)=v^{2}$, where $v \in \mathbb{F}^{\prime}(y)$. Thus $u=\left(b v^{2}-a\right) /\left(v^{2}-1\right)$ and we may takke $\sqrt{[Q(u) /-27]}=(b-a) v /\left(v^{2}-1\right)$. From $(6.6)$ it follows that (6.7)

$$
\theta=-2\left\{\left[\left(b v^{2}-a\right)((a-b) v+a-a)\right] /(v-1)(v+1)^{2}\right\}^{1 / 3}
$$

belongs to $7^{6}(y)$. If $2 \alpha \neq a+b$, the rational function in braces in (6.7) is in its lowest terms and so has no cube root in $F^{6}(y)$ for any $v(y)$ by Lemma 3.1. Indeed, even if $2 a=a+b$, then $a \neq b$ and we would require $\left(b v^{2}-a\right) /(v+1)^{2}$ to have a cube root in $F^{6}(y)$ which again contradicts Lemma 3.1 since $a \neq b$. Fence $Q(u)$ is not square-free.

(c) $\mathscr{L}_{1}, \mathscr{L}_{2}$ divide $\mathscr{D}_{f}$ with $\mathbb{Z}\left(\mathscr{L}_{1}\right)=\{2,2\}, \mathbb{E}\left(\mathscr{L}_{2}\right)=\{1,3\}$. Taking $\mathscr{L}_{2}=\mathscr{L}_{\infty}$ and proceeding with the usual normalization process we may assume that

$$
f(x)=\left(x^{2}+3 a-3\right)^{2} / 4:(2 x+3),
$$

where $a\left(\in f^{\prime}\right) \neq 1$ (otherwise $f$ is not in its lowest terms). Put $u=g(y)$. When $\infty$ is replaced by $x+2 u-2$ in the eubic resolvent of $f(x)-\imath$, we obtain

$$
\mathscr{S}(x)=x^{3}+48\left(u-(\alpha-1)^{2}\right) x-64\left(u^{2}+3(\alpha-1) u-2(\alpha-1)^{3}\right) .
$$

Thus

$$
D_{f}(u)=D_{\mathscr{S}}(u)=-27.2^{12} u^{2}\left[(u+3 a-1)^{2}-4 a^{3}\right]=-27.2^{12} u^{2} Q(u),
$$

say. Now, if $\alpha=0$, we have case (b) again. So assume that $\alpha \neq 0$, thus $Q(u)$ is square-freo. Put $u=R^{-1}\left(R^{2}+\alpha^{3}\right)-(3 \alpha-1)$. Then $Q(u)$ $=\left(R^{2}-\alpha^{\mathrm{a}}\right)^{2} / R^{2}$. If $\theta$ is given by $(6.1)$ with $\mathscr{R}=\mathscr{S}$ and $\sqrt{Q(u)}=\left(R^{2}-\alpha^{3}\right) / R$, we find that

$$
\theta=4\left(R-\alpha^{2}\right) R^{-2 / 3} .
$$

Suppose that $q \equiv 1(\bmod 3)$ so that $\sqrt{-3} \in F$. We require $\theta \in F^{3}(y) \backslash$ $F(y)$ and $u, \sqrt{D_{f}(u)} \in \mathbb{F}(y)$, whence $R=\mu S^{3}$, where $\mu$ is a non-cube in $F$ and $S \in F^{\prime}(y)$. Thus $f$ and $g$ are determined by (v) of Theorem 2.1 (II).

Alternatively, suppose that $q \equiv-1(\bmod 3)$. For $u$ and $\sqrt{D_{f}(u)}$ to be in $F(y)$ wo require $R$ in $F^{\mathbb{2}}(y)$ and $R \bar{R}=\alpha^{3}$ (where $\vec{R}$ is the conjugate of $R$ over $W^{\prime}(y)$ ). T'ogether with the fact that $\theta$ (given by (6.8)) is in $Z^{6}(y) \backslash$ $F^{2}(y)$, this implies that $R=v a^{3 / 2}(S+\sqrt{-3})^{3} /(S-\sqrt{-3})^{3}$, where $S \in F(y)$

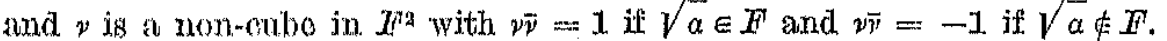
This gives tho socond part of (v) of Theorem 2.1 (II). Once again the steps are revorsible.

Note finally in this case that

$$
\mathscr{D}_{j^{*}}=\left\{\begin{array}{lll}
\left.\mathscr{L}_{1}^{2} \mathscr{L}_{2}^{2} \mathscr{L}_{\mathcal{Q}} \mathscr{L}_{4},{ }^{2}\right) & \text { if } & \sqrt{\alpha} \in F \\
\mathscr{L}_{1}^{2} \mathscr{L}_{2}^{2} \mathscr{L}, & \text { if } & \sqrt{\alpha} \notin F^{2}
\end{array}\right.
$$

(2) If $\alpha=1$, thion $R_{3}=\mathscr{L}_{1}$ (caso (a)). 
(d) $\mathscr{D}_{f}=\mathscr{L}^{2} \mathscr{Q}^{2}$. By Proposition 6.1 (ii), even in $\mathbb{H}^{2}$ we hivve $V(g)$ $\subseteq V(f)$. Moreover, in $F^{2}, \mathscr{D}_{f}=\mathscr{L}^{2} \mathscr{L}_{1}^{2} \mathscr{L}_{2}^{2}$, swy. So, by (b) and (c), there exist $L, I_{1}, L_{2}$ in $E^{2}(x)$ such that, if $f^{*}(x)=\left(x^{4}+4 x^{3}\right) / \mu(8 x-4)$ (where $\mu$ is a noncube in $\left.F^{2}\right)$ and $g^{*}(x)=x^{3}$, then

$$
f=L \circ f^{*} \circ L_{1}, \quad g=L \circ g^{*} \circ L_{1} .
$$

Now $f, g, \mu f^{*}$ and $g^{*}$ aro actually in $F(x)$. Oonsequently, (6.9) yickls

$$
f^{*}=L^{*} \circ\left(\mu \bar{\mu}^{-1}\right) f^{*} \circ L_{1}^{*}, \quad g^{*}=L^{*} \circ g^{*} \circ I_{2}^{*},
$$

whore $L^{*}=L^{-1} \circ \tilde{L}, L_{i}^{*}=\widetilde{L}_{i} \circ L_{i}^{-1}, i=1,2$ and, typienlly, $\widetilde{L}_{\text {is }}$ is the anjugate of $L$ over $F^{\prime}(x)$. It follows from $(6.10)$ that, in $7^{\prime 2}, V\left(g^{*}\right)=V\left(T^{*}\left(g^{*}\right)\right)$. However, $\left|F^{2}\right| \equiv 1(\bmod 3)$ and so $g^{*}(x) \quad\left(=x^{3}\right)$ is not a pormutation polynomial in $\mathbb{H}^{2}$. For large $q$, the only other possibility permitted by the orem 2.1 (with $m=3$ ) is that $L^{*}\left(x^{3}\right)=\left(L_{3}(x)\right)^{3}$ for some $L_{3}$ in $I^{2}$. Olcarly this implies that $L^{*}(x)=\beta x$ or $1 / \beta x$ for some non-zero $\beta$ in $x^{2}$. But then, from (6.10) again, either $f^{*}=\gamma f^{*}\left(I_{1}^{*}\right)$ or $f^{*}=1 / \gamma f^{*}\left(L_{1}^{*}\right)$, where $\gamma=\beta \mu \bar{\mu}^{-1}$. It is a simple exercise to show that tine latter alternative is impossible for any $L_{1}^{*}$ and the former implies that $L_{1}^{*}$ is the identity and $\gamma=1$. Thus $L_{1}$ and $L_{4}$ (where $L_{4}(x)=L(x / \mu)$ ) are actually in $F(x)$. However, by $(6.9)$, $f=L_{4} \circ\left(\mu f^{*}\right) \circ L_{1}$ which implies that over $F, f$ and $\mu f^{*}$ have the same muification data which by case (b) contradicts the assumption that $\mathscr{D}_{f}=\mathscr{L}^{2} \mathscr{Q}^{2}$, Hence this form is impossible.

(e) $\mathscr{D}_{f}=\mathscr{2}^{2} \mathscr{L}_{1} \mathscr{L}_{2}$ or $\mathscr{2}_{1}^{2} \mathscr{Q}_{2}$. Using Proposition 6.2 (ii) to work in $\mathbb{1}^{12}$, we have $\mathscr{D}_{f}=\mathscr{L}_{3}^{2} \mathscr{L}_{4}^{2} \mathscr{L}_{1} \mathscr{L}_{2}$, where necessarily $\mathbb{D}\left(\mathscr{L}_{3}\right)=\mathbb{E}\left(\mathscr{L}_{4}\right)=\{1,3\}$. But this is impossible by case (c).

(f) $\mathscr{D}_{f}=\mathscr{C}^{2}$. Wo must have $E(\mathscr{C})=\{1,3\}$. Replace $\mathbb{F}$ by $\mathbb{F}^{6}$ so that now $\sqrt{-3} \in \mathbb{F}$ and $\mathscr{D}_{f}=\mathscr{L}_{1}^{2} \mathscr{L}_{2}^{2} \mathscr{L}_{3}^{2}, \mathbb{E}\left(\mathscr{L}_{i}\right)=\{1,3\}, i=1,2,3$. Then although $V(g) \subseteq V(f)$ will now be false, we still must have $\sqrt{D_{f}(u)}$ (where $u=g(y)$ ) in $F(y)$. Further, as in case (b), $f=L \circ f^{*} \circ L_{1}$, whore $f^{*}(x)$ $=\left(x^{4}+4 x^{3}\right) /(4 x+a)$ and $\theta$ (givon by (6.6)) is in $F(y)$. The argument of case (b) forces $\alpha=-2$. But then $\mathbb{E}\left(\mathscr{L}_{3}\right)$ (sty) must bo $\{2,2\}$ and we have a contradiction.

This exhansts the possibilities for $\mathscr{D}_{f}$. Fence the disenssion of Theorem 2.1. for $\operatorname{deg} f=4$ is complete in the "irreducible cense".

7. Functions of degree 4 , the reducible case. We rnay suppose that $h(x, y)$ has degree 4 in $x$ and is reducible yet does not have a linear factor. Thus $h$ must be the product of two irreducible quadratics. We use troposition 1.1 in the following form.

PROPOSTron 7.1. Suppose that, in the situation of Proposition 1.1, $h=h_{1} h_{2}$, where both $h_{1}$ and $h_{2}$ are irreducible quadratics in $x$ over $I f(y)$. Then (1.1) holds if and only if $D_{h_{1}}(y) / D_{h_{2}}(y)$ is a non-square in $F$ itself.
Proof. Here (1.1) is equivalent to the fact that $h_{1}$ and $h_{2}$ have different splitting fields over $F(y)$ but the same splitting field over $F^{2}(y)$. The result follows.

Now take $h(x, y)=f(x)-g(y)$. In our situation the next assertion is not hard to see and, in any case, follows from a result of Fried (Proposition 2 of [7]). It is that there exist rational functions $\hat{f}, \hat{g}, f_{1}, g_{1}$ in $F(x)$ such thati $f=\hat{f}\left(f_{1}\right), g=\hat{g}\left(g_{1}\right), \hat{f(x)}-\hat{g}(y)$ is also the product of two irreducible factors in $F(x, y)$ and the splitting field of $\hat{f(x)}-t$ over $W(t)$ (where $t$ is an indeterminate) is the same as that of $\hat{g}(x)-t$ over $F(t)$. Clearly, deg $\hat{f}=2$ or 4 . We consider each case in turn and determine preciscly when (1.1) or its equivalent in Proposition 7.1 is satisfied.

(a) deg $\hat{f}=2$. Clearly, $\hat{g}=\hat{f}(R)$ for some $R$ in $F^{\prime}(x)$. Replacing $g_{1}$ by $R\left(g_{1}\right)$ we may assume that $\hat{g}=\hat{f}$. Further, replacing $f_{1}$ by $L \circ f_{1} \circ L_{1}$ and $\hat{f}$ by $\hat{f}\left(L^{-1}\right)$ for appropriate $L, L_{1}$ in $F^{\prime}(x)$, we may take $f_{1}(x)=x^{2}$ or $\left(x^{2}-\lambda\right) / x$, where, as always, $\lambda$ is a non-square in $F$. Indeed, we may then replace $\hat{f}$ by $L_{2}(\hat{f})$, say, and assume that $\hat{f(x)}=x^{2}+\alpha x(\alpha \in F)$ or $\hat{f(x)}=\left(x^{2}+\alpha\right) / 2(x+\beta)$ $\left(\alpha, \beta \in F\right.$, not both 0 and $\left.\beta^{2} \neq \alpha\right)$. However, if, for instance, $f(x)=x^{2}+\alpha x, f_{1}(x)=x^{2}$, then

$$
f(x)-g(y)=\left(x^{2}-g_{1}(y)\right)\left(x^{2}+g_{1}(y)+\alpha\right)
$$

and cloarly, by Proposition 7.1, (1.1) can hold only if $\alpha=0$ and $\sqrt{-1} \notin F$, i.e. $q \equiv-1$ (mod 4). In this way, it is a straightforward exercise to reduce the possibilities to one of the following (i)-(iv).

$$
\text { (i) } \hat{f}(x)=x^{2}=f_{1}(x), q \equiv-1(\bmod 4) \text {. Fere }
$$

$$
f(x)-g(y)=\left(x^{2}-g_{1}(y)\right)\left(x^{2}+g_{1}(y)\right)
$$

and (1.1) holds for any $g_{1}$ by Proposition 7.1. Moreover, $V(g)=V(f)$ if and only if for all $x$ in $F$, either $g_{1}(y)=x^{2}$ or $g_{1}(y)=-x^{2}$ is soluble for $y$ in $F$, i.e. if and only if $g_{1}=P$.

(ii) $\hat{f}(x)=\left(x^{2}+\lambda\right) / x, f_{1}(x)=x^{2}$. Here

$$
f(x)-g(y)=\left(x^{2}-g_{1}(y)\right)\left(x^{2} g_{1}(y)-\lambda\right),
$$

giving rise (as in (i)) to the pair (ii) of Theorem 2.3 .

(iii) $\hat{f}(x)=\left(x^{2}+\lambda\right) / 2 x, f_{1}(x)=\left(x^{2}-\lambda\right) / 2 x$. Fere

$$
f(x)-g(y)=\left(x^{2} g_{1}(y)-2 \lambda x_{1}-\lambda g_{1}(y)\right)\left(\infty^{2}-2 x g_{1}(y)-\lambda\right),
$$

the first quadratic having discriminant $4 \lambda\left(g_{1}^{2}+\lambda\right)$ and the second $4\left(g_{1}^{2}+\lambda\right)$. This leads to pair (iii) of Theorem 2.3. Further, $V(g)=V(f)$ if and only if for all $x$ in $F$ either $g_{1}(y)=2 \lambda x /\left(x^{2}-\lambda\right)$ or $g_{1}(y)=\left(x^{2}-\lambda\right) / 2 x$ is soluble. But, oasily,

$$
V\left(2 \lambda x /\left(x^{2}-\lambda\right)\right) \cup V\left(\left(x^{2}-\lambda\right) / 2 x\right)=F
$$


so $V(g)=V(f)$ if and only if $g_{1}=P$.

(iv) $\hat{f}(x)=x^{2}, f_{1}(x)=\left(x^{2}-\lambda\right) / x$. Here

$$
f(x)-g(y)=\left(x^{2}-x g_{1}(y)-\lambda\right)\left(x^{2}+x g_{1}(y)-\lambda\right),
$$

the two factors having identical discriminents so that Proposition 7.1 eannot be satisfied.

(b) $\operatorname{deg} \hat{f}=4$. Here $f_{x}=L$ and we may assumo, in fact, that $f=\hat{f}$. Let $K$ be the cormmon splitting field. of $f(x)-t$ and $\hat{g}(x)-t$ over $f^{\prime}(t)$ with corresponding isomorphic galois groups $G(f), G(\hat{g})$, rospectivaly. Let $y$ bo a zero of $g(x)-t$ and put $v=g_{1}(y)$. Thus $\hat{g}(v)=t$ and so $v \in K$. By Proposition 7.1, $[K(y): \not F(y)]=4$ (although $\left[X(y): F^{2}(y)\right]=2$ ). By the theorem of natural irrationalities, $r$ is divisible by 4 . But $|Q\langle\hat{g}\rangle|=r \not t(\hat{g})$ where $r \mid(H(\hat{g})-1) !$. On the other hand, $|G(f)| 24$. The only consistent conclasion is that $r=4, \operatorname{deg} \hat{g}=6$ and $G(f)=S_{4}$, the symmotric group. Thus $G(\hat{g})$ must be a transitive subgroup of $S_{6}$ isomorphic to $S_{4}$. The situation just described seems unlikely; nevertheless there are circumstances where it would occur save for the assumption that $f(x)-\hat{g}(y)$ be reducible, namely when $\hat{g}(x)$ is $\mathscr{R}\left(x^{2}\right)$, where $\mathscr{R}$ is the cubic resolvent of $f$. However, the additional hypothesis that $f(x)-\hat{g}(y)$ be reducible enables us to reach a contradiction as follows. Consider the subgroup $V$ of $G(f)$ whose members fix a prescribed root of $f(x)=t$. Then $V \cong S_{3}$. Regarding $V$ as a subgroup of $G(\hat{g})$ and using the fact that $f(x)-\hat{g}(v)$ is reducible, we see that for suitable numboring of the roots of $\hat{g}(x)=t$ we have

$$
V=\{(123)(456),(132)(465),(12)(34),(13)(4.6),(23)(56),(1)\} .
$$

However, there is no way $V$ could be one of precisely four conjugate subgroups of any transitive subgroup of $S_{6}$. So this case is, after all, impossible.

It may be helpful to point out that, in the above, the known examplo [4] of a pair $(f, g)$. with $f(x)-g(y)$ reducible and $\operatorname{deg} \hat{f}=4$ (namely, $f(x)$ $\left.=\left(x^{2}-1\right)^{2}, g(x)=-4 x^{2}\left(x^{2}-1\right)\right)$ is oliminated by the demand that $[K(y): \mathbb{F}(y)]=4$.

8. $x$-soluble polynomials of total degree 3. For generul polynomials $h(x, y)$ of degree 3 or 4 in $x$, the normalisation proceduro achioves in $\$ 5-6$ for the case $h(x, y)=f(x)-g(y)$ is not arailable. However, wo can characterise those polynomials $h$ of total degree 3 in $F[x, y]$ which are w-soluble, thus extending work of Mordell [15]. We use Proposition 5.1 in the following form.

LIMMNA 8.1. Suppose that in Proposition 5.1, $h$ has the form

$$
h(x, y)=x^{3}+h_{1}(y) x+h_{0}(y), \quad h_{0} \neq 0 .
$$

Then (5.1) holds if and only if $q \equiv-1(\bmod 3)$ and $h_{1}=0$ or

$$
h_{1}=-3\left(A^{2}+3 \lambda B^{2}\right), \quad h_{0}=2 A\left(A^{2}+3 \lambda B^{2}\right),
$$

where $A, B(\neq 0) \in F^{\prime}(y)$

Proof. If $h_{1}=0$, then $D_{h}=-27 h_{0}^{2}$ and (5.1) holds if and only if $\sqrt{-3} \notin F$.

If $h_{1} \neq 0$, put $h_{2}=-2 h_{1} A / 3$. Then

$$
D_{h}(y)=12 h_{1}^{2}\left(-\frac{1}{3} h_{1}-A^{2}\right)
$$

and (5.1) holds if and only if $-\frac{1}{3} h_{1}-A^{2}=3 \lambda B^{2}$. The result follows.

Before stating our theorem, we note that if $h$ is $x$-soluble, then so is

$$
h_{1}(x, y)=a h(b x+o y+d, e y+f), \quad a b e \neq 0,
$$

and we say that $h$ and $h_{1}$ are $x$-equivalent.

THEorem 8.2. Let $h(x, y)$ in $F[x, y]$ be a polynomial of total degree 3 and suppose that $q>q_{0}$ (absolute) and $p>3$. Then $h$ is $x$-soluble if and only if it has a faotor linear in $x$ or is $x$-equivalent to a polynomial of one of the following types:

I. $x^{3}-g(y)$,
II. $(x+y+1)^{3}-27 x y$, with $q=-1(\bmod 3)$,

III. $x^{3}+3 \eta x+y\left(3 x^{2}+\eta\right), \quad$ with $\eta=\{1$, if $q \equiv-1(\bmod 3)$, IV. $\left.x^{3}+3 \eta(y+1)^{2} x+y\left(3 x^{2}+\eta(y+1)^{2}\right),\right\}$ with $\eta= \begin{cases}1, & \text { if } q=1(\bmod 3), \\ \lambda, & \end{cases}$

V. $x^{3}-(3 x-2)\left(3 \lambda y^{2}+1\right)$.

Remark. Actually, apart from II, all the above $b$-soluble $h$ derive from functions of the form $f(x)-g(y)$. For essentially III is $[(x+\sqrt{\eta}) /(x-\sqrt{\eta})]^{3}-(y-\sqrt{\eta}) /(y+\sqrt{\eta})$, the transformation $x \rightarrow x /(y+1)$, $y \rightarrow y /(y+1)$ sends III onto IV and V is $\left(L \circ f^{*} \circ L_{1}\right)(x)-L\left(g^{*}(y)\right)$, where $f^{*}$ and $g^{*}$ are given by (i) of Theorem 2.1 (II), $L(x)=4 / x$ and $L_{1}(x)=-2 / x$.

Proof. Suppose that $h(x, y)$ is $x$-soluble. From Theorem 4.1 we may assume that $h$ is irreducible of degree 3 in $x$ and so is $x$-equivalent to a polynomial of the form (8.1) also of total degree 3. By Lemma 8.1, either $h$ is $x$-equivalent to $I$ for some $g$ or is $x$-equivalent to

$$
x^{8}-3 C^{-2}\left(A^{2}+3 \lambda B^{2}\right) x+2 A O^{-3}\left(A^{2}+3 \lambda B^{2}\right)
$$

where $A, B, C$ and the coefficients of (8.3) are all non-zero polynomials in $F[y]$. Wo may suppose also that $A, C$ and $E=A^{2}+3 \lambda B^{2}$ are co-prime. Since $C^{2} \mid Z$, then $A$ and $C$ are co-prime and so $O^{3} \mid E$. However, $A$ and $B$ are also co-prime, for, if not, we would have $A$ linear and both $A B^{-1}$ and $O$ in $F$. But then

$$
A^{-3} C^{8} h\left(A O^{-1} x, y\right)=x^{3}-3 \alpha x+2 \alpha, \quad \alpha=1+3 \lambda A^{-2} B^{2} \in F,
$$

which has discriminant $\lambda(18 B / A)^{2}$ and so is reducible, whence $h$ is reducible. 
Now write (8.3) als $x^{3}-3 G G x+2 A G$, where $O$ and $G:-O^{-3} D \in H^{\prime}(y)$ and $\operatorname{deg} \theta+\operatorname{deg} \theta \leqslant 2, \operatorname{deg} A+\operatorname{deg} G \leqslant 3$. We consider tho varions possibjlities for $C$ and $G$. Tet $\vec{F}$ be the algebraic closuro of $F$ and put $\delta=\sqrt{-3 \lambda}$ in $\bar{F}$.

(a) $\operatorname{deg} G=0$. Thus $\operatorname{deg} O=1$ or 2 and $\operatorname{deg} B=3$ or 6 . Since $A$ and $B$ are co-prime, then so are $A+\delta B$ and $A-\delta B$. Therefore, in $\overparen{F}(y), A+\delta B$ $=O_{1}^{3}$ where $O_{1}$ divides $O$. But then, since $Q \in I F, h(x, y)$ has a lactor in $\bar{F}[x, y]$ of $x+G^{1 / 3} \sigma_{1}+O G^{2 / 3} / \sigma_{1}$ (by Cardan's Tormula) which oontradicts the finct that $h$ is absolutely irreducible (Proposition 1.2 (iii)).

(b) $\operatorname{deg} \theta=\operatorname{deg} \theta=1$. This case is impossiblo for it would inply that $E$ has degree 4 yet is divisible by $O^{3}$.

(c) $\operatorname{deg} O=0, \operatorname{deg} Q=1$. For this $\operatorname{deg} A=\operatorname{deg} B=\operatorname{dog} D=1$. so that $\delta \in \mathbb{I}$, i.e. $q=-1(\bmod 3)$. Replacing $x$ by $U^{-1} x$ any $y$ by $a y+b$ for suitable $a(\neq 0), b$ in 7 , we may take $A(y)=y, B(y)=(y+1) / \sqrt{ }-3$, so that $h$ is $x$-equivalent to $x^{3}+(3 x-2 y)(2 y+1)$. Now the transformation $x \rightarrow-\frac{1}{3}(x+y+1), y \rightarrow-\frac{1}{2}(y+1)$ shows that $h$ is $x$-oquivalent to IT.

(d) $\operatorname{deg} O=0, \operatorname{deg} \theta=2$. Then $\operatorname{deg} A=2$ and $\operatorname{deg} A \leqslant 1$. If $\operatorname{deg} A$ $=\operatorname{deg} B=1$, then, as in (c), we may set $A(y)=y, B(y)=y+1$ and $h$ is 0 -equivalent to

$$
x^{3}-3\left(y^{2}+-3 \lambda(y+1)^{2}\right) x+2 y\left(y^{2}+3 \lambda(y+1)^{2}\right) .
$$

A further transtormation $x \rightarrow x+y$ indicates that $h$ is $x$-equivalent to IV but with $\eta=-3 \lambda\left(=\delta^{2}\right)$. To get $\eta=1$ in the case $q=-1$ (mod 3 ), apply the extra transformation $x \rightarrow \delta x, y \rightarrow \delta(y+1)-1$.

A similar discussion reveals that if $\operatorname{deg} A=1, \operatorname{deg} B=0$, then $h$ is $x$-equivalent to IIx, while if $\operatorname{deg} A=0, \operatorname{deg} B=1$, then $h$ is a-equitwalent to $\mathrm{V}$.

The sufficiency of $\mathrm{I}-\mathrm{V}$ is obvious from the above and Lemma 8.1. Thus the proof is complete.

From Theorem 8.2 , it is easy to guess which cubics $h$ are both $n$-soluble and $y$-soluble in $F$. Formal verification of Mordoll's result [15] (stated below) is indeed possible from this starting point and probably represents a shorter and less intricate method than that of Mordell. Novorticlests, the proof is not actually irmmediato and, for brevity, is omitited.

Thwordim 8.3 (Mordell). Let $h(x, y)$ in $T^{5}[x, y]$ have total degree 3 . Suppose that $g>g_{0}$ and $p>3$. Then $h$ is both $x$-soluble and $y$-soluble in $I I$ if and only if $h$ has a factor linear in $x$ and a factor (possibly the same) linear in $y$ or one of $h(x, y)$ and $h(y, x)$ is of the form (8.2) with $0=0$, whene $h_{1}$ is one of I-III in Theorem 8.2 with $g(y)=y$ or $y^{3}+1$. in $\mathrm{T}$.

9. Covering sets. We shall call a sot of tunctions $\left\{f_{i}(x)\right\}$ in $I^{\prime}(x)$ a 00 vering set if $\bigcup V\left(f_{i}\right)=F$. There are some simple examples. (i) $\left\{f_{i}\right\}, f_{i}=P$ for some $i$.

(ii) $\left\{x^{r}, \gamma x^{r}, \ldots, \gamma^{r-1} x^{r}\right\}$, where $r \mid(q-1)$ and $\gamma$ in $F$ is a non $a$ th power for any divisor $d$ of $r$ ith $d>1$.

(iii) $\left\{\left(x^{2}-\lambda\right) / 2 x, 2 \lambda x /\left(x^{2}-\lambda\right)\right\}$ (see $\S 7$ ).

Using exponential sums, Mordcll [16], [17], has constructed a nontrivial covering set comprising a function of degree 4 and a function of dogree 3. However, the natural approach to covering sets may be to use the following result which follows immediately from Proposition 1.1. (Fou rolated work on tho more gencral problem $V(f) \subseteq \bigcup_{i} V\left(f_{i}\right)$, see [5], $[9], \$ 4$.

PRopostrion 9.1. In the situation of Proposition 1.1, let

$$
h(x, y)=\prod_{i=1}^{m}\left(f_{i}(x)-y\right) .
$$

Suppose that

$$
G^{*}(K, F(y))=\bigcup_{i=1}^{m}\left(\bigcup_{x_{i}} G^{*}\left(K, F\left(x_{i}\right)\right)\right)
$$

where the inner union is over all roots $x_{i}$ of $f_{i}(x)=y$. Then $\left\{f_{i}\right\}$ is a oovering set for $F$.

Using Proposition 9.1 and previous results in this paper; we can demonstrate some examples of covering sets valid for any $F$ with $p>3$.

(iv) Mordell's covering set

$$
\left\{f_{1}, f_{2}\right\}_{1}=\left\{x^{4}+a x^{2}+b x,\left(x^{3}+2 a x^{2}-a^{2} x-b^{2}\right) / 4 x\right\}, \quad b \neq 0,
$$

follows easily from Proposition 9.1 since $f_{2}(x)-y$ is the cubic resolvent of $f_{1}(x)-y$. Another covering set arising in this way is

$$
\left\{\left(x^{4}+a x+b\right) / x^{2}, x-\left(a^{2} /\left(x^{2}-4 b\right)\right)\right\}, \quad a b \neq 0 .
$$

(v) From (i) of Theorem 2.1 (II) (ef. Theorem 8.2 (v)), we get the pair

$$
\left\{\left(x^{3}-3 x+2\right) /(3 x-2), 3 x^{2}\right\} \text {. }
$$

(vi) From (iii) of Theorem 2.1. (II) we get the pair

$$
\left\{f_{1}, f_{2}\right\}=\left\{\left(x^{4}+4 x^{3}\right) /(8 x-4), x^{3}\right\} .
$$

Howover, atthough this is a covering set for all $q$, the manner of the covering depouds on $q$. For, of course, if $q=-1$ (mod 3 ), then $f_{2}=P$ and we have a trivial eovering set of type (i). On the other hand, if $q=1$ (mod 3), then $\left|V\left(f_{1}\right) \cap V\left(f_{2}\right)\right| \div q / 12$. Put

(vii) Finally, we exhibit a non-trivial covering set of three functions.

$$
\left\{f_{1}, f_{2}, f_{3}\right\}=\left\{\left(x^{2}-1\right)^{2},-4 x^{2}\left(x^{2}-1\right), \frac{7}{2}(x-1)^{2} /\left(x^{2}+1\right)\right\}
$$


(As noted earlier ( $\$ 7), f_{1}(x)-f_{2}(y)$ is reducible.) Then the roots of $f_{i}(x)$ $=y, i=1,2,3$, can be written as $\left\{\alpha_{1},-\alpha_{1}, \alpha_{2},-\alpha_{2}\right\},\left\{\beta_{1},-\beta_{1}, \beta_{2},-\beta_{2}\right\}$, $\left\{\gamma, \gamma^{-1}\right\}$, respectively, where $\beta_{i}=\frac{1}{2}\left(a_{1} \pm a_{2}\right), i=1,2$, and

$$
\gamma=-(2 y-1)^{-1}\left(1-2 \alpha_{1} \alpha_{2}\left(\alpha_{1}^{2}-1\right)\right) \text {. }
$$

Moreover, $G^{*}(K, F(y))$ is the whole galois group $\theta$, say, and has order 8 . If the roots of each $f_{i}(x)=y$ are numbered in the order given, then the action of $G$ as a permutation of these roots is as follows:

\begin{tabular}{r|r|r}
\multicolumn{1}{c|}{$f_{1}$} & \multicolumn{1}{|c|}{$f_{2}$} & \multicolumn{1}{c}{$f_{3}$} \\
\hline$(1)$ & $(1)$ & $(1)$ \\
$(12)$ & $(14)(23)$ & $(12)$ \\
$(34)$ & $(13)(24)$ & $(12)$ \\
$(12)(34)$ & $(12)(34)$ & $(1)$ \\
$(13)(24)$ & $(34)$ & $(12)$ \\
$(14)(23)$ & $(12)$ & $(12)$ \\
$(1423)$ & $(1324)$ & $(1)$ \\
$(1324)$ & $(1423)$ & $(1)$
\end{tabular}

Thus $\left\{f_{1}, f_{2}, f_{3}\right\}$ is a covering set by Proposition 9.1.

\section{References}

[1] S. D. Cohen, The distribution of polynomials over finite fields, Acta Arith. 17 (1970), pp. 255-271.

[2] - The distribution of polynomials over finite fields, IX, ibid. 20 (1972), pp. 53-62.

[3] - Oomposite rational functions whioh are powers, Proc. R. Soc. Edinburgh A, 83 A (1979), pp. 11-16.

[4] H. Davenport, D. J. Lewis and A.' Sclinzel, Equations of the form $f(x)$ $=g(y)$, Quart. J. Math. Oxford (2) 12 (1961), pp. 304-312.

[5] M. Fried, Arithmetioal properties of value sets of polynomials, Acta Arith. I.5 (1969), pp. 9l-125.

[6] - On a conjecture of Sohur, Michigan Math. J. 17 (1970), pp. 41-55.

[7] - The field of definition of function fields and a problem in the reduoilibily of polynomials in two variables, Illinois J. Math. 17 (1973), pp. 128-146.

[8] - On a thearem of MaoOhuer, Acta Arith. 25 (1974), pp. $121 \mathrm{~lm} 26$.

[0] - Arithmetical properties of funotion fielde, $I I$; The generalized Sohur problem, ibid. 25 (1974), pp. 225-258.

[10] - On Eilbert's irreducibility theorem, J. Number Theory 6 (1974), pp. 211-231.

[11] - Galois groups and oomplest multiptioation, Trans. Amer. Math. Soc. 235 (1978), pp. 141-163.

[12] N. Jacobson, Basic algebra $T$, Freoman, San Francisco 1974.

[13] I. Kaplansky, Filelds and rings, Chicago lectures in Mathomatics, Chicago 1969.
[14] K. McCann and K. S. Williams, Oubic polynomials with the same residues. $(\bmod p)$, Proc. Cambridge Phil. Soc. 64 (1968), pp. 655-658.

[15] L. J. Mordell, Oubio polynomials with the same residues (mod $p$ ), Proc. London. Math. Soc. (3) 21 (1970), pp. 129-144.

[16] - Rational funotions representing all residues $\bmod p$, J. London Math. Soc. 5 (1972), pp. 166-168.

[17] - Rational funotions representing all residues modp, II, Proc. Amer. Math. Soc. 35 (1972), pp. $411-412$

[18] G. I. Perol'muter, On certain sums of characters, Uspekhi Matem. Nank $18(1963)$, pp. $145-149$

UNIVIIISTIY OF GLABGOW

ow $80 \mathrm{~W}$

Scotland

Received on 30.8 .1978

and in revised form on 9.11 .1978

(1097) 